\title{
Virtual Endoscopy Using Cubic QuickTime-VR Panorama Views
}

\author{
Ulf Tiede ${ }^{1}$, Norman von Sternberg-Gospos ${ }^{1}$, \\ Paul Steiner ${ }^{2}$, and Karl Heinz Höhne ${ }^{1}$ \\ 1 Institute of Mathematics and Computer Science in Medicine (IMDM) \\ 2 Dept. of Radiology \\ University Hospital Hamburg-Eppendorf, Germany
}

\begin{abstract}
Virtual endoscopy needs some precomputation of the data (segmentation, path finding) before the diagnostic process can take place. We propose a method that precomputes multinode cubic panorama movies using Quick-Time-VR. This technique allows almost the same navigation and visualization capabilities as a real endoscopic procedure, a significant reduction of interaction input is achieved and the movie represents a document of the procedure.
\end{abstract}

\section{Introduction}

Virtual endoscopy (VE) and especially virtual colonoscopy (VC) have gained much attention in diagnostic radiology recently. After acquisition of a MRI or $\mathrm{CT}$ volume data set the procedure involves the following computation steps:

- segmentation of the colon

- determination of the (typically central) path through the colon

- navigation through the colon and visualisation of the colon wall

- documentation of the flight through the colon as a movie

- transfer of the document together with the report to the referring physician

- in a modern environment: archiving and communication of the document in a PACS

In clinical practice it has turned out to be advantageous to do the segmentation in advance and also to compute a path before viewing, because otherwise the navigation is extremely tedious and time consuming and needs a complex user interface. In ideal cases segmentation can be done automatically [1], however, in some cases manual control is required due to noise in the data or motion artefacts. A common approach for path computation is skeletonization 2]. For noisy data and due to wrickles in the colon highly sophisticated heuristics are needed to remove dead ends and loops, to end up with a smooth path. Another way to support navigation is described in [3]. Here a potential field is calculated, which is used to compute a force that pushes the virtual viewer back onto the central path. The strength of the force depends on the distance of the current view point to the path. At the colon wall the force is infinite thus inhibiting the 
user to pass through the wall. Other approaches unfold the inner colon surface to a $2 \mathrm{D}$ image map [3. However, clinicians are unfamiliar with these images in addition to geometric distortion problems.

The visualization of the colon wall requires a conversion of the colon surface to polygonal meshes in order to utilize standard computer graphics hardware and software, which allow to compute $3 \mathrm{D}$ views at near real-time speed for modest size datasets. Available voxel-based accelerators (e.g. Mitsubishi VolumePro) cannot perform perspective transformations directly which are essential for calculating endoscopic views 4].

Outgoing from the fact, that navigation is complex (even when a central path is already defined) and real-time visualization for high quality rendering cannot be achieved on standard PCs, we investigated an approach that also pre-computes a large part of the visualization. Expected advantages are that visualization is restricted to "meaningful" images and interaction becomes easier.

\section{Method and Material}

If we would try to include any possible view in a pre-computed movie, the amount of data would be huge and navigation would not be simple at all. If we use only a fixed field of view and a constant viewing direction while moving through the colon along the central path one might miss important details which are outside the selected field of view (e.g. behind wrinkles). A well-known technique from classical photography is the use of wide angle ("fish-eye") lenses, but these yield strong geometric distortions. Another approach that we propose here is the generation of panorama images where a number of overlapping photographs are taken while the camera is rotated around the vertical axis. The single photographs are then connected to form a cylindrical image also known as a panorama view. While this technique needs some effort in photography it can easily be simulated in computer graphics (fig. 1). However, cylindrical projections do not allow

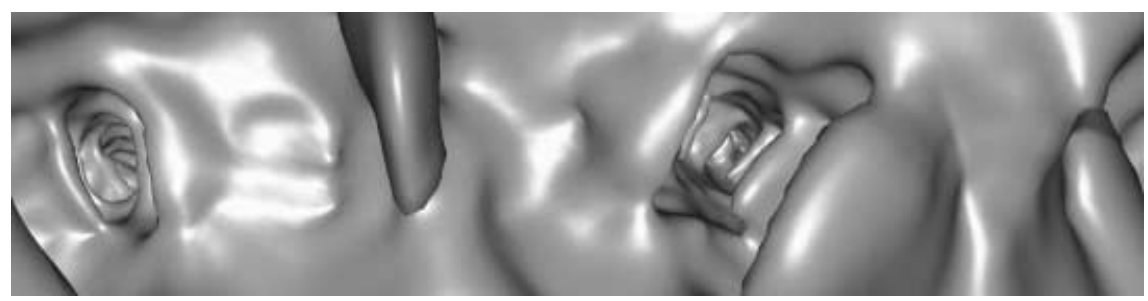

Fig. 1. Cylindrical panorama view of the colon.

straight views up and down, i.e. $90^{\circ}$ upwards or downwards from the horizon due to geometric distortions that become apparent at viewing angles larger than $45^{\circ}$. A generalization of cylindrical panoramas are spherical panoramas, which allow viewing in all directions. Here an image map is computed which is then 
"wrapped" around a sphere. This requires complex geometry calculations as the inverse problem, namely unfolding a sphere to a 2D-map, is a well-known problem in cartography. A simplification for general spherical panoramas are cubic panoramas. Cubic panoramas are computed as follows: From a given viewpoint we compute six 3D projections, which correspond to the six faces of a cube. The viewpoint is assumed to be located at the center of the cube, i.e. the first projection looks into the direction along the central path, then we turn $90^{\circ}$ to the right, compute the 2nd image. Turn again $90^{\circ}$ to the right, now we are looking back into the direction we came from, turn again and finally we compute the images when looking $90^{\circ}$ upwards and downwards. As can easily be seen the six projections must be calculated with a field of view of exactly $90^{\circ}$ so that the images fit nicely together and form the faces of a cube (fig. 2). Recently Apple Computer,

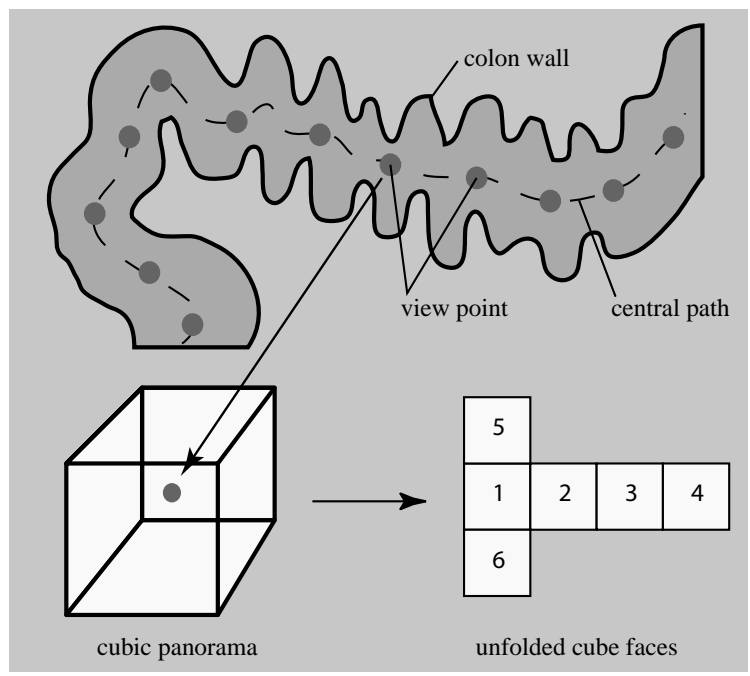

Fig. 2. Cubic panorama generation. At any viewing position 6 projections corresponding to the 6 faces of a cube are computed with a field of view of $90^{\circ}$.

Inc. has incorporated cubic panoramas into their QuickTime-VR technology [5]. The QuickTime player has a build-in distortion correction method for viewing these cubic panoramas. The six 3D projections are stored as a sequence of images together with additional information that allows QuickTime to recognize the images to be a cubic panorama. The QuickTime player then allows continuous navigation across the entire panorama in real-time using the two degrees of freedom of the mouse. For a continuous motion through the colon we compute such cubic panoramas along the central path every few millimeters with a high resolution rendering algorithm described in [6]. The single panoramas, which are called nodes in QuickTime terminology, must then be connected to enable the player to "jump" from one node to the next. This is accomplished 
using so called hot spots, which are just an additional image layer, where the pixel values correspond to the node number the player may move to. These hot spot images are very simple and can be calculated automatically for colonoscopy, because there are no bifurcations. Thus if we are at node $n$ the pixel value of the hot spot for all possible forward-looking directions, i.e. $180^{\circ}$ field of view from the initial viewing direction, is $\mathrm{n}+1$, and the value for all backward-looking directions is $\mathrm{n}-1$. The result is a multi-node cubic panorama movie. In contrast to other pre-computed movies, the amount of data is thus decisively reduced. In addition the interaction with QTVR movies is very simple.

As each panorama node has its own initial viewing parameters, i.e. pan and tilt angles and field of view, it is important for a smooth transition from one node to the next not to use these initial values to preserve and transfer the parameters of the current node. We modified the behaviour of the player to handle this requirement, so that it become possible to move through the colon while inspecting the colon wall laterally.

For diagnostic purposes it is not sufficient to provide endoscopic views only. For the radiologist, who is familiar with interpreting CT and MR images, it is a necessity to have access to the original data at any stage of the viewing process. Therefore we do not only store the panorama images but the corresponding zbuffers and the related viewing transformation matrices as well (fig. [3). With this

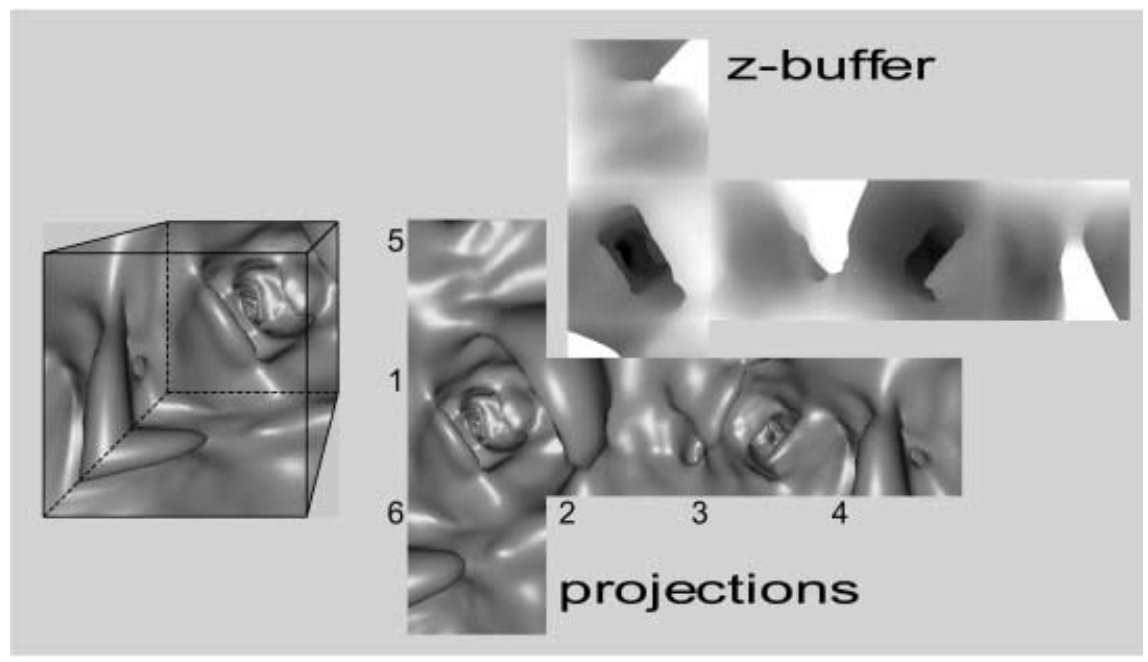

Fig. 3. Cubic panorama projections. Right: Image map of the 6 unfolded cube faces (front) and their corresponding z-buffers (rear). The distortion correction for viewing a panorama is performed automatically by the QuickTime player.

additional information available we can easily get back from any surface location into the original gray scale volume. Furthermore this allows the simultaneous 
display of different views such as 3D outside views, in which the current virtual camera position and orientation can be marked to facilitate orientation.

\section{Results}

We applied the described method to about 20 contrast enhanced MRI datasets of the colon. The datasets were segmented by thresholding and the central path as well as up to 200 high resolution cubic panoramas together with their corresponding z-buffers were computed and stored in the QuickTime-VR movie format. Additionally a matrix of 3D overview images that show the colon from outside were created to facilitate orientation. These images are stored as a QuickTimeVR object movie which is very similar to panorama movies. The radiologist can then access the final movie over the intranet on his desktop PC. He can "move" through the colon in real-time while looking around in all directions searching for abnormalities. On the 3D overview he can verify were the virtual endoscope is currently located. Also, just by clicking on the colon surface at any location, the corresponding position is marked on three orthogonal cross-sections showing the original MR values. This helps the radiologist who is familiar with $2 \mathrm{D}$ cross-sections in the assessment of suspected lesions. Fig. 4 shows the simple graphical interface we have developed for virtual colonoscopy. Our radiologists

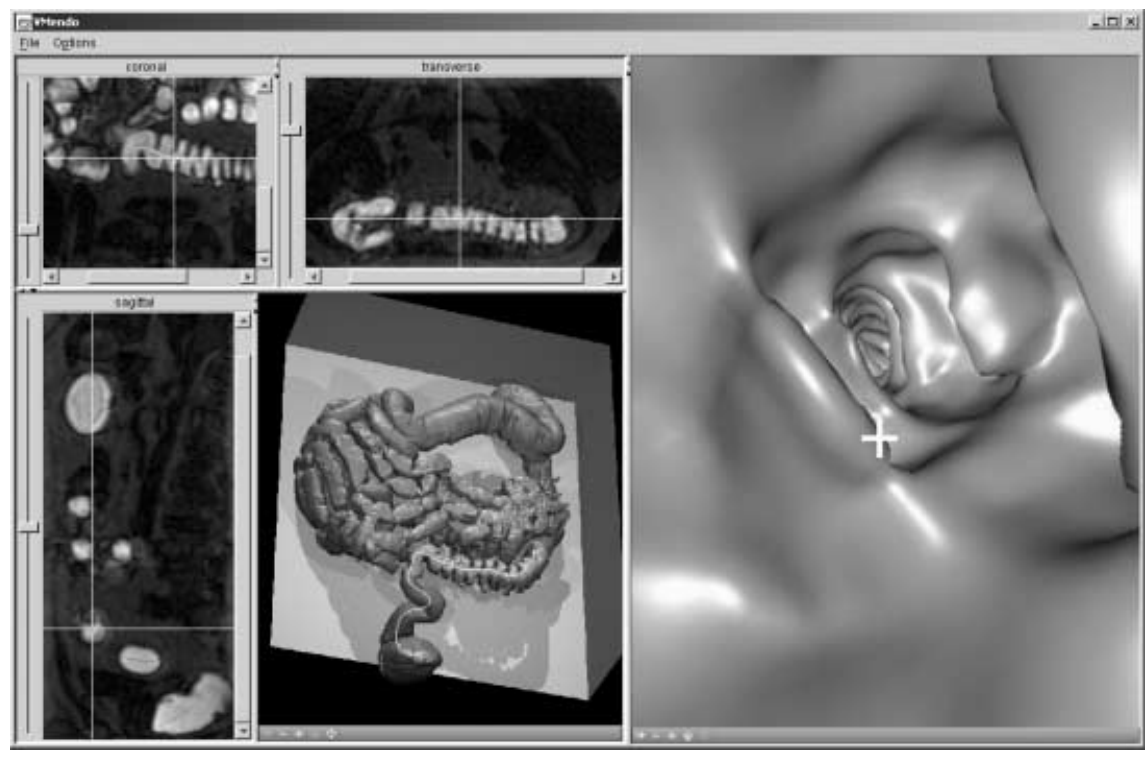

Fig. 4. User interface showing an endoscopic view (right), a 3D overview image (middle bottom) and three orthogonal MR cross-sections. The marked position on the colon wall (white cross) corresponds to the positions on the cross-sections and in the overview. 
feel comfortable with the user interface. The entire procedure takes a couple of minutes for establishing a diagnosis.

\section{Conclusion}

Using pre-computed cubic panorama images for virtual endoscopy allows quasi real-time viewing and easy navigation on a standard PC. Especially the very simple user interface has proved helpful for the acceptance of the procedure. The method can also be applied to structures with bifurcations like blood vessels or the bronchi. Fig. 5 shows as an example the bifurcation of the bronchi computed from the Visible Human data set [7]. As a decisive advantage the panorama movie

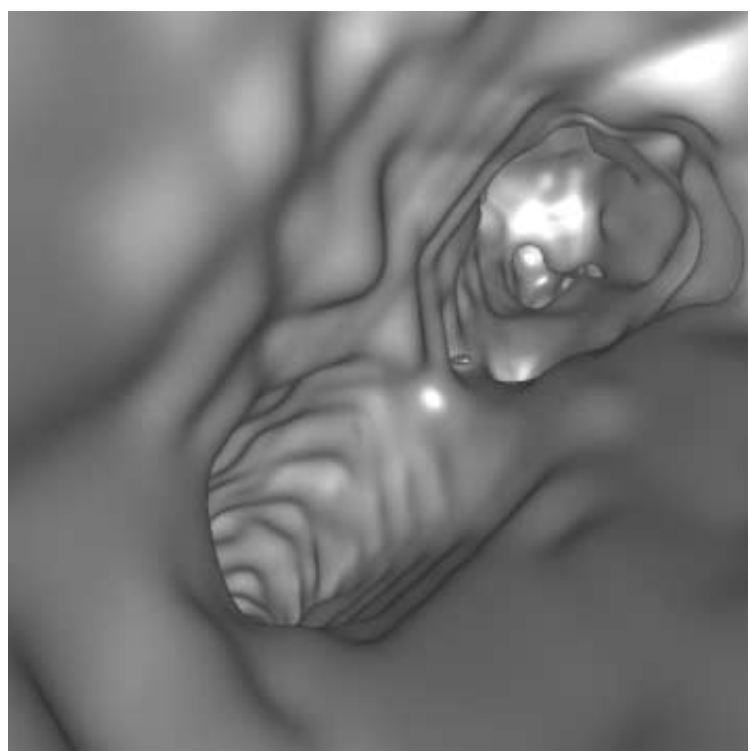

Fig. 5. Panorama view of the main bifurcation of the bronchi computed from the Visible Human Dataset.

represents a document similar to classical video recordings of real endoscopic examinations with additional functionality that can be viewed with standard software at any PC or with browser technology in an Intranet. Due to the fact that computation time is not an issue, also more sophisticated rendering methods like automatic color labeling of suspicious regions can easily be incorporated. If connected to a knowledge base [8] additional features like symbolic descriptions and manipulation capabilities become possible, which allow to generate even more comprehensive teaching and learning material [9].

Currently the number of panoramas in a single QuickTime-VR movie is limited to 255 which is not a real restriction for the application shown here. Further research should also take stereoscopic viewing into account. 


\section{References}

1. M. Sato, S. Lakare, M. Wan, A. Kaufman, Z. Liang, and M. Wax. An automatic colon segmentation for 3D virtual colonoscopy. IEICE Transactions on Information and Systems, E84-D(1):201-208, January 2001.

2. Y. Zhou and A. W. Toga. Efficient Skeletonization of Volumetric Objects. IEEE Transactions on Visualization and Computer Graphics, 5(3):196-209, 1999.

3. A. Vilanova i Bartroli, R. Wegenkittl, A. König, and E. Gröller. Nonlinear Virtual Colon Unfolding. In Mike Bailey and Charles Hanson, editors, Proc. IEEE Visualization 2001, pages 91-98, San Diego, CA, October 2001.

4. M. Wan, W. Li, K. Kreeger, I. Bitter, A. Kaufman, Z. Liang, D. Chen, and M. Wax. 3D Virtual Colonoscopy with Real-time Volume Rendering. In Chin-Tu Chen and Anne V. Clough, editors, Proc. SPIE Medical Imaging 2000, volume 3978, pages 165-171, San Diego, CA., February 2000.

5. Inc. Apple Computer. Interactive Movies: QuickTime VR.

http://developer.apple.com/techpubs/quicktime/qtdevdocs/PDF/insideqt_qtvr.pdf, 2001.

6. Ulf Tiede, Thomas Schiemann, and Karl Heinz Höhne. High Quality Rendering of Attributed Volume Data. In David Ebert, Hans Hagen, and Holly Rushmeier, editors, Proc. IEEE Visualization 1998, pages 255-262, Research Triangle Park, NC, 1998. (ISBN 0-8186-9176-X).

7. A. Pommert, K.H. Höhne, B. Pflesser, E. Richter, M. Riemer, T. Schiemann, R. Schubert, U. Schumacher, and U. Tiede. Creating a high-resolution spatial/symbolic model of the inner organs based on the Visible Human. Medical Image Analysis, 5(3):221-228, 2001.

8. Rainer Schubert, Bernhard Pflesser, Andreas Pommert, Kay Priesmeyer, Martin Riemer, Thomas Schiemann, Ulf Tiede, P. Steiner, and Karl Heinz Höhne. Interactive volume visualization using "intelligent movies". In James D. Westwood, Helene M. Hoffman, Richard A. Robb, and Don Stredney, editors, Medicine meets Virtual Reality, Proc. MMVR '99, volume 62 of Studies in Health Technology and Informatics, pages 321-327. IOS Press, Amsterdam, 1999.

9. K.H. Höhne, B. Pflesser, A. Pommert, K. Priesmeyer, M. Riemer, T. Schiemann, R. Schubert, U. Tiede, H.-C. Frederking, S. Gehrmann, S. Noster, and U. Schumacher. VOXEL-MAN 3D-Navigator: Inner Organs. Regional, Systemic and Radiological Anatomy. Springer-Verlag Electronic Media, Heidelberg, 2000. (3 CD-ROMs, ISBN 3-540-14759-4). 